\title{
A flat projective variety with $D_{8}$-holonomy Francis E. A. Johnson
}

\begin{abstract}
We show explicitly that the compact flat Kähler manifold of complex dimension three with $D_{8}$ holonomy studied by Dekimpe, Halenda and Szczepanski ([5] p. $367)$ possesses the structure of a nonsingular projective variety. This corrects a previous statement by H. Lange in [9] that the holonomy group of a hyperelliptic threefold is necessarily abelian.
\end{abstract}

The study of flat Riemannian manifolds, begun by Bieberbach [2], has subsequently acquired a very extensive literature. See, for example, [3],[4],[13],[14]. In a paper published in the Tohoku Mathematical Journal [9], H. Lange investigated closed flat manifolds of real dimension six which, in addition, possess the structure of nonsingular complex projective varieties which have finite étale coverings by abelian varieties. In Lange's terminology such varieties are called hyperelliptic three-folds.

The significant claim of Lange's paper is that the (finite) holonomy group of such a hyperelliptic three-fold is necessarily abelian. In particular, Lange claims that the dihedral group of order eight ${ }^{\dagger}$ does not occur as a holonomy group in this context.

Lange's claim is mistaken, however. In the present paper we show explicitly that the compact flat Kähler manifold of complex dimension three with $D_{8}$ holonomy studied by Dekimpe, Halenda and Szczepanski ([5] p. 367) does indeed possess the structure of a nonsingular projective variety. In fact, the existence of this complex algebraic structure was previously shown, in a very general context, by the present author in the paper [7]. However, as Lange also makes a statement which explicitly claims to contradict the main result of [7] it seems appropriate, in setting the matter straight, to give a direct, and elementary, construction of the algebraic structure whose existence Lange denies.

The present paper is organised as follows; in $\S 1$ we give a brief review of the theory of flat Riemannian manifolds as it pertains both to Kähler manifolds and projective varieties; in $\S 2$ we give a completely elementary criterion which guarantees that some flat Riemannian manifolds admit the structure of a nonsingular complex algebraic variety. Whilst this criterion does not immediately apply to the most general cases, it is quite sufficient to deal with all cases in which the holonomy group is $D_{8}$. In $\S 3$ we construct an explicit complex algebraic structure for the Kähler manifold of Dekimpe, Halenda and Szczepanski. This can be checked by direct calculation and requires very little theory beyond an appeal to the criterion of $\S 2$.

2010 MSC Primary 53C29; Secondary 14F35, 14K02, 32J27.

Keywords and phrases: Flat manifold; holonomy group; complex projective variety.

$\dagger$ In this paper we denote the dihedral group of order 8 by $D_{8}$. This would now seem to be the generally accepted convention (cf [10], [12], [13]). In Lange's notation however the dihedral group of order 8 is denoted by $D_{4}$. 
In $\S 4$, we explain the choice of complex structure made in $\S 3$ by reference to the representation theory of $D_{8}$. Finally, in $\S 5$, we give a brief summary of the general situation.

The author wishes to express his gratitude to Dieter Kotschick for drawing his attention to this matter.

\section{§1: Generalities on flat Kähler manifolds:}

Let $X$ be a closed flat Riemannian manifold of dimension $2 n$ in which $\pi_{1}(X)$ occurs in an exact sequence

$$
0 \rightarrow \mathbb{Z}^{2 n} \rightarrow \pi_{1}(X) \rightarrow \Phi \rightarrow 1
$$

with operator representation $\rho: \Phi \rightarrow G L_{2 n}(\mathbb{Z})$. Here $\Phi$ is finite and extension of scalars to $\mathbb{R}$ gives the holonomy representation of the Riemannian manifold $X$

$$
\widehat{\rho}: \Phi \rightarrow G L_{2 n}(\mathbb{R}) .
$$

In what follows we will denote by $L$ the $\mathbb{Z}[\Phi]$ module $L=\left(\mathbb{Z}^{2 n}, \rho\right)$ associated to the representation $\rho$ so that $L \otimes_{\mathbb{Z}} \mathbb{R}$ is the $\mathbb{R}[\Phi]$ module $L=\left(\mathbb{R}^{2 n}, \widehat{\rho}\right)$. We first observe the following (cf (3.1) of [7], also (3.1) of [8]):

(1.1) $X$ admits the structure of a flat Kähler manifold if and only if there exists $t \in \operatorname{End}_{\mathbb{R}[\Phi]}\left(L \otimes_{\mathbb{Z}} \mathbb{R}\right)$ such that $t^{2}=-$ Id.

Such a complex structure $t$ is then covariant with respect to the $\Phi$-action. We now regard $\left(L \otimes_{\mathbb{Z}} \mathbb{R}, \tau\right)$ as a complex vector space. The complex torus $\left(L \otimes_{\mathbb{Z}} \mathbb{R}, t\right) / L$ is then a finite unbranced covering of $X$ via the action of $\Phi$. In this connection, and as a consequence of Kodaira's Embedding Theorem, there is a general principle of isogeny (cf [6], p.192).

(1.2) Let $Z, \widehat{Z}$ be compact, connected, complex manifolds and let $p: \widehat{Z} \rightarrow Z$ be a holomorphic mapping which is topologically an unbranched covering. Then $Z$ is a nonsingular complex projective variety if and only if $\widehat{Z}$ is a nonsingular complex projective variety.

To return to a consideration of flat Kähler manifolds $X$ as above, the following is an immediate consequence of the isogeny principle:

(1.3) If the complex structure $t \in \operatorname{End}_{\mathbb{R}[\Phi]}\left(L \otimes_{\mathbb{Z}} \mathbb{R}\right)$ can be chosen so that $\left(L \otimes_{\mathbb{Z}} \mathbb{R}, t\right) / L$ is a complex algebraic torus then $X$ admits the structure of a nonsingular complex projective variety.

\section{$\S 2$ : A sufficient condition for algebraicity:}

Let $\Phi$ be a finite group. By a lattice over $\Phi$ we mean a module $L$ over $\mathbb{Z}[\Phi]$ whose underlying abelian group is finitely generated and torsion free. When $\mathbb{F}$ is either $\mathbb{Q}$ or $\mathbb{R}$ we denote the corresponding $\mathbb{F}[\Phi]$ module by $L_{\mathbb{F}}=L \otimes_{\mathbb{Z}} \mathbb{F}$. In (1.1) above 
we say that $t$ is a covariant complex structure for $L$. Notice that there is a stronger possibility, namely that it might be possible to choose $t \in \operatorname{End}_{\mathbb{Q}[\Phi]}\left(L \otimes_{\mathbb{Z}} \mathbb{Q}\right)$ such that $t^{2}=-\mathrm{Id}$. In that case we say that $t$ is a covariant $\mathbb{Q}$-complex structure for $L$. The following is elementary linear algebra:

Proposition 2.1 : Let $t: V \rightarrow V$ be an $\mathbb{Q}$-linear map satisfying $t^{2}=-\operatorname{Id}_{V}$ where $V$ is a finite dimensional vector space over $\mathbb{Q}$; then $\operatorname{dim}_{\mathbb{Q}}(V)=2 n$ and $V$ admits a basis $\left\{e_{1}, \ldots, e_{n}, f_{1}, \ldots, f_{n}\right\}$ such that $t\left(e_{r}\right)=f_{r}: t\left(f_{r}\right)=-e_{r}$.

Theorem 2.2 : Let $V$ be a vector space of dimension $2 n$ over $\mathbb{Q}$, let $t: V \rightarrow V$ be a $\mathbb{Q}$-linear map satisfying $t^{2}=-\mathrm{Id}_{V}$ and let $L \subset V$ be an additive subgroup such that $\operatorname{rk}_{\mathbb{Z}}(L)=2 n$. Then $\left(V \otimes_{\mathbb{Q}} \mathbb{R}, t \otimes \mathrm{Id}\right) / L$ is a complex algebraic torus.

Proof: Choose a basis $\left\{e_{1}, \ldots, e_{n}, f_{1}, \ldots, f_{n}\right\}$ such that $t\left(e_{r}\right)=f_{r}: t\left(f_{r}\right)=-e_{r}$ and put $\Gamma=\operatorname{span}_{\mathbb{Z}}\left\{e_{r}, f_{r}: 1 \leq r \leq n\right\}$. It is then straightforward to see that $\left(V \otimes_{\mathbb{Q}} \mathbb{R}, t \otimes \mathrm{Id}\right) / \Gamma$ is a complex algebraic torus. Now $L+\Gamma$ is also a free abelian group of rank $2 n$ so that $\left(V \otimes_{\mathbb{Q}} \mathbb{R}, t \otimes \mathrm{Id}\right) /(L+\Gamma)$ is also a complex torus. Moreover, $\Gamma$ is contained with finite index in $L+\Gamma$. As $\left(V \otimes_{\mathbb{Q}} \mathbb{R}, t \otimes I d\right) /(L+\Gamma)$ is finitely covered by the algebraic torus $\left(V \otimes_{\mathbb{Q}} \mathbb{R}, t \otimes \mathrm{Id}\right) / \Gamma$ it follows from $(1.2)$ that $\left(V \otimes_{\mathbb{Q}} \mathbb{R}, t \otimes \mathrm{Id}\right) /(L+\Gamma)$ is also a complex algebraic torus. Finally as $L$ also imbeds as a subgroup of finite index in $L+\Gamma$ it follows that the complex torus $\left(V \otimes_{\mathbb{Q}} \mathbb{R}, t \otimes \mathrm{Id}\right) / L$ is a finite covering of the complex algebraic torus $\left(V \otimes_{\mathbb{Q}} \mathbb{R}, t \otimes \mathrm{Id}\right) /(L+\Gamma)$. It now follows from (1.2) that $\left(V \otimes_{\mathbb{Q}} \mathbb{R}, t \otimes \mathrm{Id}\right) / L$ is also algebraic.

In the context of flat manifolds we now have:

Theorem 2.3 : Let $X$ be a flat Riemannian manifold with holonomy group $\Phi$ and holonomy module $L=\left(\mathbb{Z}^{2 n}, \rho\right)$; if $L$ admits a covariant $\mathbb{Q}$-complex structure $t$ then $X$ admits the structure of a nonsingular complex projective variety.

Proof : For then $\left(L \otimes_{\mathbb{Z}} \mathbb{R}, t \otimes I d\right) / L$ is a complex algebraic torus and the conclusion follows from (1.3).

\section{$\S 3$ : The example of Dekimpe, Halenda and Szczepanski:}

In their paper ([5] p. 367) Dekimpe, Halenda and Szczepanski construct a flat Kähler manifold of complex dimension three with holonomy group $D_{8}$. In this example the holonomy group is the subgroup of $\mathrm{GL}_{6}(\mathbb{Z})$ generated by

$$
A_{1}=\left(\begin{array}{rrrrrr}
1 & 0 & 0 & 0 & 0 & 0 \\
0 & 0 & 0 & -1 & 0 & 0 \\
0 & 0 & -1 & 0 & 0 & 0 \\
0 & -1 & 0 & 0 & 0 & 0 \\
0 & 0 & 0 & 0 & -1 & 0 \\
0 & 0 & 0 & 0 & 0 & -1
\end{array}\right) ; A_{2}=\left(\begin{array}{rrrrrr}
0 & 0 & 1 & 0 & 0 & 0 \\
0 & 1 & 0 & 0 & 0 & 0 \\
1 & 0 & 0 & 0 & 0 & 0 \\
0 & 0 & 0 & -1 & 0 & 0 \\
0 & 0 & 0 & 0 & -1 & 0 \\
0 & 0 & 0 & 0 & 0 & -1
\end{array}\right)
$$




\section{A flat projective variety}

To verify that this example is indeed Kähler then by (1.1) above it is sufficient to find $t \in G L_{6}(\mathbb{R})$ such that

i) $t^{2}=-\mathrm{I}_{6}$ and ii) $t A_{i}=A_{i} t$ for $i=1,2$.

Observe that condition ii) is simply the requirement that the complex structure $t$ is an endomorphism of the holonomy module. To show, in addition, that this example is a complex algebraic manifold it is sufficient, by (2.3), to find such a complex structure $t$ which also has the property that

iii) $t \in G L_{6}(\mathbb{Q})$.

One may check by direct calculation that

$$
t=\frac{1}{2}\left(\begin{array}{rrrrrr}
0 & 1 & 0 & -1 & 0 & 0 \\
-2 & 0 & -2 & 0 & 0 & 0 \\
0 & 1 & 0 & 1 & 0 & 0 \\
2 & 0 & -2 & 0 & 0 & 0 \\
0 & 0 & 0 & 0 & 0 & 2 \\
0 & 0 & 0 & 0 & -2 & 0
\end{array}\right)
$$

satisfies conditions i), ii) and iii). Consequently we see that:

Theorem 3.2: The flat Kähler manifold with $D_{8}$-holonomy constructed in [5] admits the structure of a nonsingular complex projective variety which is finitely covered by an abelian variety.

\section{$\S 4$ : Representations of $D_{8}$ :}

Whilst the properties of the complex structure of $\S 3$ can be checked directly with a minimum of theory, its choice is by no means arbitrary. In this section we explain its construction within the context of the representation theory of $D_{8}$.

Write

$$
D_{8}=\left\langle x, y: x^{4}=1 ; y^{2}=1, y x=x^{3} y\right\rangle .
$$

We single out five indecomposable $\mathbb{Z}\left[D_{8}\right]$-lattices, denoted by $S, T, U, V, W$ and defined by the following integral representations: $S$ has rank 2 over $\mathbb{Z}$ and is given by

$S: \quad x \mapsto\left(\begin{array}{rr}0 & 1 \\ -1 & 0\end{array}\right) \quad ; \quad y \mapsto\left(\begin{array}{rr}0 & -1 \\ -1 & 0\end{array}\right)$

$T, U, V, W$ are all rank 1 , and given as follows:

$$
\begin{array}{lll}
T: & x \mapsto 1 ; & \mapsto \mapsto 1 ; \\
U: & x \mapsto-1 ; & y \mapsto 1 ; \\
V: & x \mapsto 1 ; & \mapsto \mapsto-1 ; \\
W: & x \mapsto-1 ; y \mapsto-1 .
\end{array}
$$


We note the that Wedderburn decomposition of $\mathbb{Q}\left[D_{8}\right]$ is

$$
\mathbb{Q}\left[D_{8}\right] \cong M_{2}(\mathbb{Q}) \times \mathbb{Q} \times \mathbb{Q} \times \mathbb{Q} \times \mathbb{Q}
$$

Thus there are five isomorphically distinct simple $\mathbb{Q}\left[D_{8}\right]$-modules and it is straightforward to see that these are isomorphic to $S_{\mathbb{Q}}, T_{\mathbb{Q}}, U_{\mathbb{Q}}, V_{\mathbb{Q}}, W_{\mathbb{Q}}$. In what follows we will need only $S_{\mathbb{Q}}$ and $T_{\mathbb{Q}}$. However, we note also the indecomposable module $\widetilde{S}$ of rank 2 over $\mathbb{Z}$ defined by

$\widetilde{S}$

$$
x \mapsto\left(\begin{array}{rr}
0 & 1 \\
-1 & 0
\end{array}\right) \quad ; \quad y \mapsto\left(\begin{array}{rr}
1 & 0 \\
0 & -1
\end{array}\right) .
$$

Whilst $\widetilde{S}$ is not isomorphic to $S$ as a $\mathbb{Z}\left[D_{8}\right]$-module, it becomes isomorphic to $S$ as a module over $\mathbb{Q}\left[D_{8}\right]$. In fact, putting $P=\left(\begin{array}{rr}1 & 1 \\ -1 & 1\end{array}\right)$ we calculate that

$$
P\left(\begin{array}{rr}
0 & 1 \\
-1 & 0
\end{array}\right)=\left(\begin{array}{rr}
0 & 1 \\
-1 & 0
\end{array}\right) P \text { and } P\left(\begin{array}{rr}
1 & 0 \\
0 & -1
\end{array}\right)=\left(\begin{array}{rr}
0 & -1 \\
-1 & 0
\end{array}\right) P \text {. }
$$

Thus we have :

(4.2) The correspondence $\mathbf{v} \mapsto P \mathbf{v}$ defines an isomorphism $P: \widetilde{S}_{\mathbb{Q}} \stackrel{\simeq}{\longrightarrow} S_{\mathbb{Q}}$.

The construction of the complex structure $t$ of $\S 3$ is more perspicuous if instead we use an alternative system of generators for the holonomy group. Thus put $X=A_{1} A_{2}$ and $Y=A_{1}$ so that

$$
X=\left(\begin{array}{rrrrrr}
0 & 0 & 1 & 0 & 0 & 0 \\
0 & 0 & 0 & 1 & 0 & 0 \\
-1 & 0 & 0 & 0 & 0 & 0 \\
0 & -1 & 0 & 0 & 0 & 0 \\
0 & 0 & 0 & 0 & 1 & 0 \\
0 & 0 & 0 & 0 & 0 & 1
\end{array}\right) \quad ; \quad Y=\left(\begin{array}{rrrrrr}
1 & 0 & 0 & 0 & 0 & 0 \\
0 & 0 & 0 & -1 & 0 & 0 \\
0 & 0 & -1 & 0 & 0 & 0 \\
0 & -1 & 0 & 0 & 0 & 0 \\
0 & 0 & 0 & 0 & -1 & 0 \\
0 & 0 & 0 & 0 & 0 & -1
\end{array}\right)
$$

One checks that $X^{4}=\mathrm{Id} ; Y^{2}=\mathrm{Id} ; Y X=X^{3} Y$. The correspondences $\eta(x)=X ; \eta(y)=Y$ then describe the holonomy module $\left(\mathbb{Z}^{6}, \eta\right)$. Put

$$
Q=\left(\begin{array}{llllll}
1 & 0 & 0 & 0 & 0 & 0 \\
0 & 0 & 1 & 0 & 0 & 0 \\
0 & 1 & 0 & 0 & 0 & 0 \\
0 & 0 & 0 & 1 & 0 & 0 \\
0 & 0 & 0 & 0 & 1 & 0 \\
0 & 0 & 0 & 0 & 0 & 1
\end{array}\right)
$$


and define $\rho(g)=Q \eta(g) Q^{-1}$. Then $\rho$ decomposes into block $2 \times 2$ form as follows

$$
\rho(x)=\left(\begin{array}{ccc}
\rho_{+}(x) & 0 & 0 \\
0 & \rho_{-}(x) & 0 \\
0 & 0 & \rho_{0}(x)
\end{array}\right) \quad ; \quad \rho(y)=\left(\begin{array}{ccc}
\rho_{+}(y) & 0 & 0 \\
0 & \rho(y)_{-} & 0 \\
0 & 0 & \rho_{0}(y)
\end{array}\right)
$$

where $\rho_{+}(x), \rho_{+}(y), \rho_{-}(x), \rho_{-}(y)$ are given by

$$
\begin{gathered}
\rho_{+}(x)=\left(\begin{array}{rr}
0 & 1 \\
-1 & 0
\end{array}\right) ; \rho_{+}(y)=\left(\begin{array}{rr}
1 & 0 \\
0 & -1
\end{array}\right) \\
\rho_{-}(x)=\left(\begin{array}{rr}
0 & 1 \\
-1 & 0
\end{array}\right) ; \rho_{-}(y)=\left(\begin{array}{rr}
0 & -1 \\
-1 & 0
\end{array}\right) \\
\rho_{0}(x)=\left(\begin{array}{ll}
1 & 0 \\
0 & 1
\end{array}\right) ; \rho_{-}(y)=\left(\begin{array}{rr}
-1 & 0 \\
0 & -1
\end{array}\right) .
\end{gathered}
$$

That is, the correspondence $\mathbf{v} \mapsto Q \cdot \mathbf{v}$ defines an isomorphism of $\mathbb{Z}\left[D_{8}\right]$-modules

$$
Q:\left(\mathbb{Z}^{6}, \eta\right) \stackrel{\simeq}{\longrightarrow} \widetilde{S} \oplus S \oplus T \oplus T .
$$

Now put $H=(P \oplus \operatorname{Id} \oplus \operatorname{Id} \oplus \operatorname{Id}) \circ Q$; then $H$ defines an isomorphism of $\mathbb{Q}\left[D_{8}\right]$-modules

$$
H:\left(\mathbb{Q}^{6}, \eta\right) \stackrel{\simeq}{\longrightarrow} S_{\mathbb{Q}} \oplus S_{\mathbb{Q}} \oplus T_{\mathbb{Q}} \oplus T_{\mathbb{Q}} .
$$

However, the $\mathbb{Q}\left[D_{8}\right]$-module $S_{\mathbb{Q}} \oplus S_{\mathbb{Q}} \oplus T_{\mathbb{Q}} \oplus T_{\mathbb{Q}}$ admits an obvious covariant $\mathbb{Q}$-complex structure $\theta$ which we write in block form as follows

$$
\theta=\left(\begin{array}{rrrr}
0 & \mathrm{I}_{2} & 0 & 0 \\
-\mathrm{I}_{2} & 0 & 0 & 0 \\
0 & 0 & 0 & 1 \\
0 & 0 & -1 & 0
\end{array}\right)
$$

Consequently the holonomy module $\left(\mathbb{Z}^{6}, \eta\right)$ admits the covariant $\mathbb{Q}$-complex structure

$$
t=H^{-1} \circ \theta \circ H
$$

Straightforward calculation now shows that $t$ has the form specified in (3.1). 
$\S 5$ : The general theory of covariant complex structures:

When $\Phi$ is a finite group the rational group ring $\mathbb{Q}[\Phi]$ is semisimple and so has a Wedderburn decomposition as a product of matrix rings over division algebras thus:

$$
\mathbb{Q}[\Phi]=M_{d_{1}}\left(D_{1}\right) \times \cdots \times M_{d_{m}}\left(D_{m}\right) .
$$

Moreover the canonical involution $c: \mathbb{Q}[\Phi] \rightarrow \mathbb{Q}[\Phi]$ induced by $c(g)=g^{-1}$ is positive in the sense that $0<\operatorname{Tr}(\alpha c(\alpha))$ for any nonzero element $\alpha \in \mathbb{Q}[\Phi]$. We single out four classes of division algebras $D$ of finite dimension over $\mathbb{Q}$; here $\mathbb{E}$ and $\mathbb{K}$ are fields of finite degree over $\mathbb{Q}$.

I: $\quad D=\mathbb{E}$ is totally real;

II: $\quad D=\left(\frac{a, b}{\mathbb{E}}\right)$ where $\mathbb{E}$ is totally real, $a$ is totally positive, $b$ is totally negative;

III: $\quad D=\left(\frac{a, b}{\mathbb{E}}\right)$ where $\mathbb{E}$ is totally real, $a$ and $b$ are totally negative;

IV : $D=(\mathbb{K}, s, a)$, where $s$ is an automorphism of $\mathbb{K}$ whose fixed point field $\mathbb{E}$ is an imaginary quadratic extension, $\mathbb{E}=\mathbb{E}_{0}(\sqrt{b})$ of a totally real field $\mathbb{E}_{0}$ and $a \in \mathbb{E}$; moreover, if $\mathbb{F}$ is a maximal totally real subfield of $\mathbb{E}$, there exists a totally positive element $d \in \mathbb{F}$ such that $N_{\mathbb{E} / \mathbb{E}_{0}}(a)=N_{\mathbb{F} / \mathbb{E}_{0}}(d)$.

It is a consequence of Albert's classification [1] of positively involuted semisimple algebras over $\mathbb{Q}$ that in the Wedderburn decomposition (5.1) each division algebra $D_{i}$ is restricted to one of the above types I, II, III, IV. If $V$ is a simple $\mathbb{Q}[\Phi]$ module then its endomorphism ring $\operatorname{End}_{\mathbb{Q}[\Phi]}(V)$ is isomorphic to one of the division algebras $D_{1}, \ldots, D_{m}$ occuring in the Wedderburn decomposition (5.1). If $\mathrm{T}$ denotes one of the types I, II, III, IV we say that $V$ is of type T when $\operatorname{End}_{\mathbb{Q}[\Phi]}(V)$ is of type T. Theorem 3.3 of [7] can now be expressed as follows:

(5.2) If $L$ is a lattice over $\mathbb{Q}[\Phi]$ then the following conditions on $L$ are equivalent;

(i) $\quad L_{\mathbb{R}}$ admits a covariant complex structure $\theta$;

(ii) $\quad$ each $\mathbb{Q}[\Phi]$-simple module of type I has even multiplicity in $L_{\mathbb{Q}}$;

(iii) $\quad L_{\mathbb{Q}}$ admits a covariant complex structure $t$ such that $\left(L_{\mathbb{R}}, t\right) / L$ is an algebraic complex torus.

It should perhaps be stressed that the complex structures $\theta$ and $t$ need not be the same. The statement (i) of (5.2) is an existential statement within the real representation theory of $\Phi$. However, it implies the much stronger statement (ii) which is a precise quantative statement within the rational representation theory of $\Phi$. For the holonomy group $D_{8}$, as the foregoing calculations show, once (ii) is granted the required complex structure is constructed in a quite elementary fashion. For more general holonomy groups however, the construction of the complex structure $t$ making $\left(L_{\mathbb{R}}, t\right) / L$ into an abelian variety requires a more detailed application of representation theory in the manner of Albert [1] and Shimura [11]. 
There is a special case of (5.2) in which a covariant complex structure $t$ is especially easy to construct, namely when the rational Wedderburn decomposition has the form

$$
\mathbb{Q}[\Phi] \cong M_{d_{1}}(\mathbb{Q}) \times \ldots \times M_{d_{m}}(\mathbb{Q}) .
$$

To achieve a Kähler structure it is then necessary that every simple $\mathbb{Q}[\Phi]$-module occurs with even (possibly zero) multiplicity in the rational holonomy module $L_{\mathbb{Q}}$, so that $L_{\mathbb{Q}} \cong V_{\mathbb{Q}} \oplus V_{\mathbb{Q}}$ for some $\mathbb{Q}[\Phi]$-module $V_{\mathbb{Q}}$. Then

$$
t=\left(\begin{array}{cc}
0 & \mathrm{Id}_{V} \\
-\mathrm{Id}_{V} & 0
\end{array}\right)
$$

is a covariant $\mathbb{Q}$-complex structure for $L$. As we have seen, this is the case when $\Phi=D_{8}$. It is also the case when $\Phi=\mathcal{S}_{n}$, the symmetric group on $n$ letters. In view of (1.3), we obtain the following as a consequence of (5.2):

Theorem 5.3: Let $X$ be a closed flat Riemannian manifold of dimension $2 n$. If $X$ admits the structure of a flat Kähler manifold then $X$ also admits the structure of a nonsingular complex projective variety which is finitely covered by an abelian variety.

Again it should be stressed that the initial complex structure $\theta$ on $X$ posited in the statement that $X$ admits a Kähler structure need not be the same as the final complex structure $t$ which renders $X$ as a complex algebraic manifold. What can be said, however, is that given the existential statement that $X$ admits a Kähler structure then the construction of a complex structure endowing $X$ with structure of a nonsingular complex projective variety can be accomplished in a straightforward manner.

In this context let us now examine the example given by Lange on p.501 of [9]. Lange considers an elliptic curve $B_{1}$ on which the cyclic group $C_{4}$ acts freely and holomorphically, and also a complex torus $B_{2}$ on which $C_{4}$ acts via the matrix

$$
\left(\begin{array}{cc}
i & \alpha \\
0 & -i
\end{array}\right)
$$

where $\alpha$ can be any complex number. We shall write $X(\alpha)$ for the quotient manifold $X(\alpha)=C_{4} \backslash\left(B_{1} \times B_{2}\right)$ As Lange correctly points out, $X(\alpha)$ is a flat Kähler manifold and in general fails to be an algebraic manifold. Lange claims that this example contradicts the main theorem of [7]. However, this claim is incorrect.

To see this, first note that the flat Kähler manifold $X(0)$ is algebraic. Moreover

$$
\Gamma\left(\begin{array}{cc}
i & \alpha \\
0 & -i
\end{array}\right) \Gamma^{-1}=\left(\begin{array}{cc}
i & 0 \\
0 & -i
\end{array}\right) \text { where } \Gamma=\left(\begin{array}{cc}
1 & -\frac{i \alpha}{2} \\
0 & 1
\end{array}\right)
$$

Consequently the mapping $\left(\mathbf{b}_{1}, \mathbf{b}_{2}\right) \mapsto\left(\mathbf{b}_{1}, \Gamma \cdot \mathbf{b}_{2}\right)$ induces an affine diffeomorphism $\Gamma_{*}: X(\alpha) \stackrel{\simeq}{\longrightarrow} X(0)$ of the underlying real flat manifolds. Thus in accordance with the main theorem of [7], the flat Riemannian manifold represented by the nonalgebraic Kähler structure $X(\alpha)$ also possesses the structure of a complex algebraic manifold, namely $X(0)$. 


\section{A flat projective variety}

\section{References}

[1] A. A. Albert, Involutorial simple algebras and real Riemann matrices, Ann. of Math. 36 (1935), 886-964.

[2] L. Bieberbach, Über die Bewegungsgruppen der Euklidischen Raume I, Math. Ann. 70 (1911), 297-336.

[3] L. S. Charlap, Compact flat Riemannian manifolds I, Ann. of Math. 81 (1965), 15-30.

[4] L. S. Charlap, Bieberbach groups and flat manifolds, Springer Verlag. 1986.

[5] K. Dekimpe, M. Halenda and A. Szczepanski, Kähler flat manifolds, J. Math. Soc. Japan. 61 (2) (2009), 363-377.

[6] P. Griffiths and J. Harris, Principles of Algebraic Geometry, John Wiley and Sons, 1978.

[7] F. E. A. Johnson, Flat algebraic manifolds, Geometry of lowdimensional manifolds I (Durham 1989), 73-91, London Math. Soc. Lecture Note Ser. 150, Cambridge Univ. Press, Cambridge 1990.

[8] F. E. A. Johnson and E. G. Rees, Kähler groups and rigidity phenomena, Math. Proc. Camb. Phil. Soc. 109 (1991), 31-44.

[9] H. Lange, Hyperelliptic varieties, Tohoku Math. J. 53 (2001), 491-510.

[10] J. Milnor, Groups which act on $S^{n}$ without fixed points, Amer. J. Math. 79 (1957), 623-630.

[11] G. Shimura, On analytic families of polarised abelian varieties and automorphic functions, Ann. of Math. 78 (1963), 149-192.

[12] R. G. Swan, Projective modules over binary polyhedral groups, Journal für die Reine und Angewandte Mathematik. 342 (1983), 66-172.

[13] A. Szczepanski, Geometry of crystallographic groups, World Scientific, 2012.

[14] J. A. Wolf, Spaces of constant curvature: McGraw-Hill, 1967.

F.E.A. Johnson

Department of Mathematics

University College London

Gower Street, London WC1E 6BT, U.K.

e-mail address : feaj@math.ucl.ac.uk 